\title{
Modelagem Matemática e Simulação Computacional da Dinâmica de um Robô SCARA
}

\author{
Josias Guimarães Batista ${ }^{1}$ \\ Instituto Federal de Ciência e Tecnologia do Ceará, Depto de Tecnologia Da Indústria. \\ Campus Tabuleiro do Norte, CE 377 - km 2, Sítio Taperinha - 62.960-000 Tabuleiro do \\ Norte - CE
}

\section{José Leonardo Nunes da Silva ${ }^{2}$}

Instituto Federal de Ciência e Tecnologia do Ceará, Depto de Tecnologia Da Indústria. Campus Limoeiro do Norte, Rua Estevam Remígio 1145, CEP 62930-000, Limoeiro do Norte-CE

\section{Nícolas Silva Pereira ${ }^{3}$}

Universidade Federal do Ceará, Depto de Eng. De Teleinformática - Campus do Pici, s/n, Bloco 725, CEP 60455-900, Fortaleza-CE

\section{George André Pereira Thé ${ }^{4}$}

Universidade Federal do Ceará, Depto de Eng. De Teleinformática - Campus do Pici, s/n, Bloco 725, CEP 60455-900, Fortaleza-CE

Resumo. Este trabalho apresenta a modelagem matemática de um robô SCARA de três juntas e a simulação computacional da dinâmica. Para a simulação foi elaborada uma trajetória em hélice que descreve o deslocamento do robô e por ultimo são apresentados os valores de posição, velocidade, aceleração e torque de cada junta.

Palavras-chave. Robô, SCARA, Modelagem, Cinemática, Dinâmica.

\section{Introdução}

Com o crescimento do consumo mundial a ampliação da produção se fez necessária, no intuito de buscar uma solução para suprir esta necessidade, as indústrias de modo geral utilizarem robôs no lugar da mão de obra humana para muitas tarefas insalubres [6].

Robótica é uma área complexa que envolve muitas áreas do conhecimento como física, ciências dos materiais, eletrônica, controle, processamento de sinais, programação e manufatura $[2,3]$.

Neste trabalho é apresentada a modelagem matemática de um robô manipulador tipo SCARA (Seletiva Compliant Assembléia Robot Arm). O robô aqui apresentado

\footnotetext{
1 josias.batista@ifce.edu.br, josiasgb@yahoo.com.br

2 leonardo.silva@ifce.edu.br, jleonardonunes@gmail.com

${ }^{3}$ nicolassilva.ti@gmail.com

${ }^{4}$ george.the@ufc.br, geothe@ hotmail.com
} 
encontra-se fora de operação por problemas no controlador, mas toda mecânica e motores encontram-se em perfeito estado de funcionamento. A modelagem inclui a cinemática (direta, inversa e diferencial) e dinâmica, além das simulações realizadas no software MATLAB. A figura 1 abaixo mostra o robô SCARA o qual se referencia esse trabalho. Por último mostra-se o resultado da posição, velocidade, aceleração e torque de cada junta obtidos a partir de uma trajetória helicoidal. O robô SCARA foi montado, pelos autores, no Centro de Automação e Robótica (CENTAURO) da UFC.

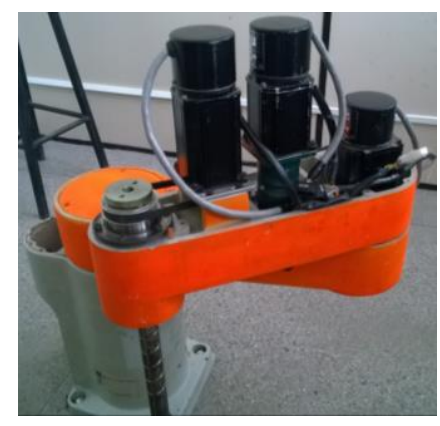

Figura 1: Robô SCARA.

\section{Modelagem Computacional}

Com o objetivo de obter informações relevantes do braço robótico foi feita uma modelagem computacional do mesmo no Solid Edge, como mostra a figura 2. Observando as dimensões reais e o material do braço (ferro e alumínio fundido).

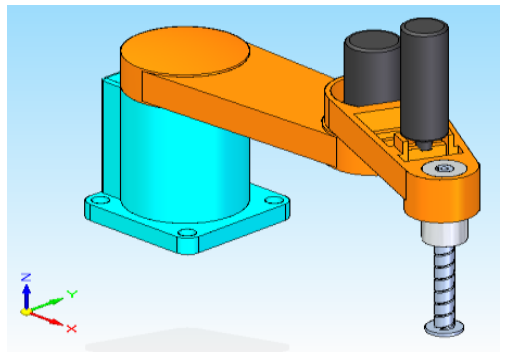

Figura 2: Estrutura do robô SCARA.

A partir da modelagem computacional foram obtidos: a massa de cada peça do braço, centro de massa de cada etapa da montagem e matriz dos tensores de inércia. As figuras 3 e 4, abaixo, têm-se o exemplo do primeiro elo do robô e as propriedades físicas do mesmo.

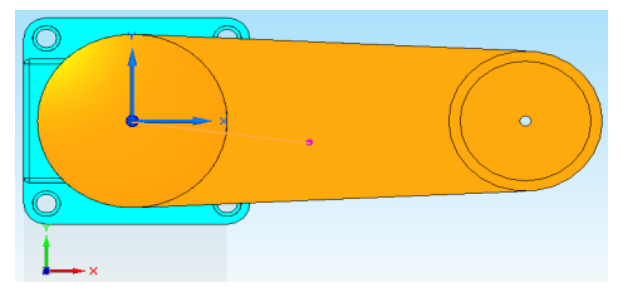

Figura 3: Modelagem do primeiro elo. 


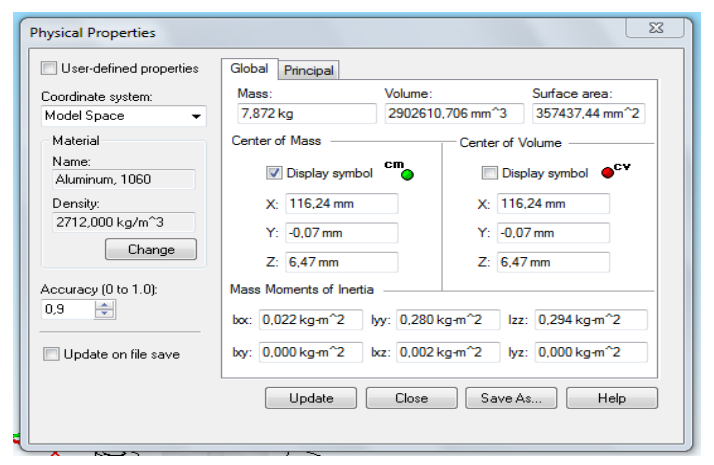

Figura 4: Propriedades físicas do primeiro elo.

Para os demais elos foi aplicado o mesmo procedimento do primeiro elo.

\section{Modelagem Matemática do robô SCARA}

A modelagem matemática de robôs industriais é geralmente dividida em modelagem cinemática e dinâmica $[1,5]$.

\subsection{Cinemática direta}

As equações da cinemática direta do robô SCARA foram encontradas a partir da alocação dos sistemas de referencias e aplicação dos parâmetros D-H [7, 8, 10]. Com isso são obtidas as matrizes de transformação de junta e em seguida calcula-se a matriz de transformação homogênea, visto em (1), que indica a localização do elemento final do robô, a ferramenta, em relação ao sistema de referencia da base.

$$
T={ }_{0}^{1} H \cdot{ }_{1}^{2} H \cdot{ }_{2}^{3} H \cdot{ }_{3}^{4} H={ }_{0}^{4} H
$$

Qualquer posição do elemento final do robô pode ser encontrada no espaço de tarefa a partir das coordenadas no espaço das juntas como observado abaixo em (2).

$$
\left[\begin{array}{c}
P_{x} \\
P_{y} \\
P_{z}
\end{array}\right]=\left[\begin{array}{c}
l_{2} C_{1}+l_{3} C_{12} \\
l_{2} S_{1}+l_{3} S_{12} \\
l_{1}-q_{3}
\end{array}\right],
$$

\subsection{Cinemática inversa}

Para resolver o problema da cinemática inversa adotou-se o método geométrico e foram encontrados os seguintes parâmetros abaixo:

$$
\theta_{1}=\tan ^{-1}\left(\frac{y}{x}\right)-\tan ^{-1}\left(\frac{l_{2} \sin \theta_{2}}{l_{1}+l_{2} \cos \theta_{2}}\right)
$$




$$
\begin{gathered}
\theta_{2}= \pm \cos ^{-1}\left(\frac{x^{2}+y^{2}-\left(l_{1}^{2}+l_{2}^{2}\right)}{2 l_{1} l_{2}}\right) \\
q_{3}=l_{1}-P_{z}
\end{gathered}
$$

\subsection{Cinemática diferencial}

Segue, em [6], a matriz Jacobiana do robô SCARA obtida da modelagem da cinemática do mesmo [9] :

$$
\left[\begin{array}{c}
\dot{x} \\
\dot{y} \\
z \\
\omega_{x} \\
\omega_{y} \\
\omega_{z}
\end{array}\right]=\left[\begin{array}{ccc}
-\left(l_{1} \sin \theta_{1}+l_{2} \sin \left(\theta_{1}+\theta_{2}\right)\right) & -l_{2} \sin \left(\theta_{1}+\theta_{2}\right) & 0 \\
l_{1} \cos \theta_{1}+l_{2} \cos \left(\theta_{1}+\theta_{2}\right) & l_{2} \cos \left(\theta_{1}+\theta_{2}\right) & 0 \\
0 & 0 & -1 \\
0 & 0 & 0 \\
0 & 0 & 0 \\
1 & 1 & 0
\end{array}\right] \cdot\left[\begin{array}{c}
\dot{\theta}_{1} \\
\dot{\theta}_{2} \\
\dot{d}_{3}
\end{array}\right]
$$

\subsection{Dinâmica}

A dinâmica do manipulador foi modelada usando a mecânica Lagrangeana e utilizando o espaço das juntas [9].

A equação geral adotada neste trabalho que descreve a dinâmica do manipulador está escrita como [9]:

$$
\tau=D(q) \ddot{q}+C(q, \dot{q}) \dot{q}+G(q)
$$

As matrizes acima são dadas por:

$$
\begin{aligned}
\mathrm{D}(\mathrm{q}) & =\left|\begin{array}{ccc}
2.25 C_{2}+5.92 & 1.13 C_{2}+2.73 & 0 \\
1.13 C_{2}+2.73 & 2.73 & 0 \\
0 & 0 & 4.5
\end{array}\right| \\
C(q) & =\left|\begin{array}{ccc}
-1.13 \dot{q}_{2} S_{2} & -1.13\left(\dot{q}_{1}+\dot{q}_{2}\right) S_{2} & 0 \\
1.13 \dot{q}_{1} S_{2} & 0 & 0 \\
0 & 0 & 0
\end{array}\right| \\
G(q) & =\left|\begin{array}{c}
16.48 C_{12}+50.95 C_{1} \\
16.48 C_{12} \\
-44,15
\end{array}\right|
\end{aligned}
$$




\section{Métodos}

Para executar a simulação do robô foi elaborado um algoritmo que descrevesse uma trajetória, figura 5, em hélice, que simula o deslocamento de cada junta.

Espaço Cartesiano

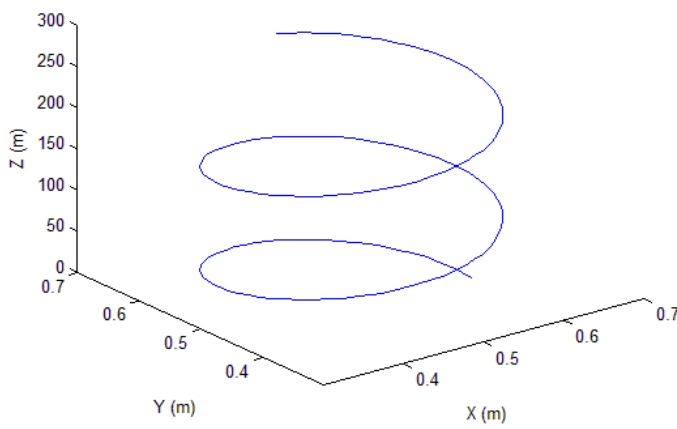

Figura 5: Trajetória helicoidal executada pelo robô.

\section{$5 \quad$ Resultados}

Como a aplicação da trajetória helicoidal descrita pelo robô, pode-se obter o comportamento da posição, velocidade, aceleração e torque de cada junta mostrado nas figuras $6,7,8$ e 9 , respectivamente.
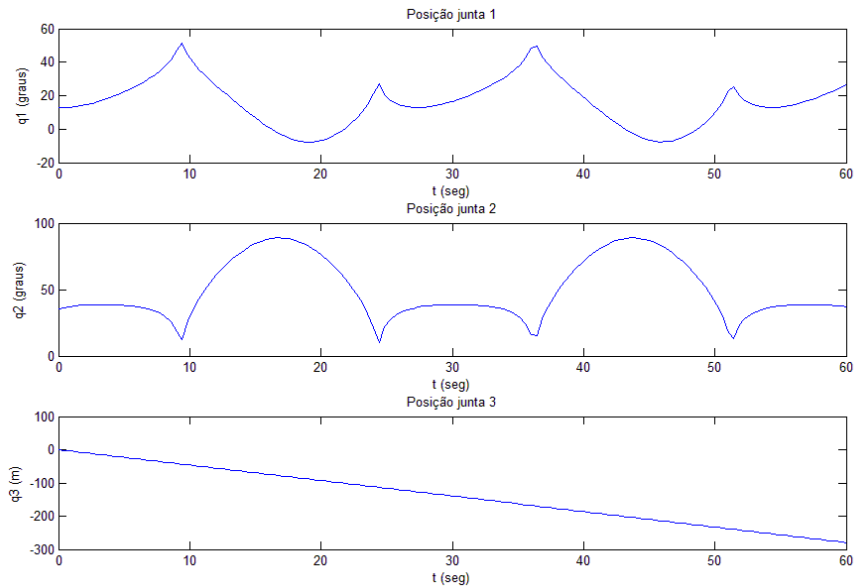

Figura 6: Posições de cada junta 

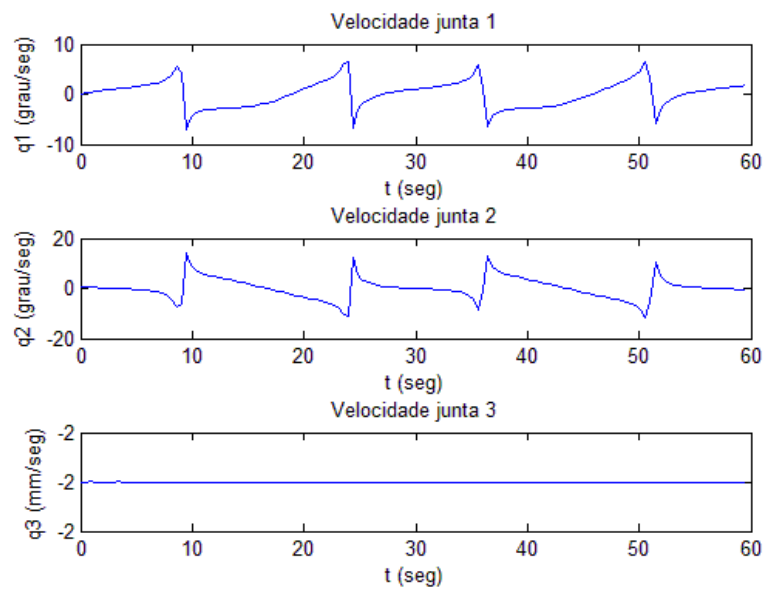

Figura 7: Velocidades de cada junta.
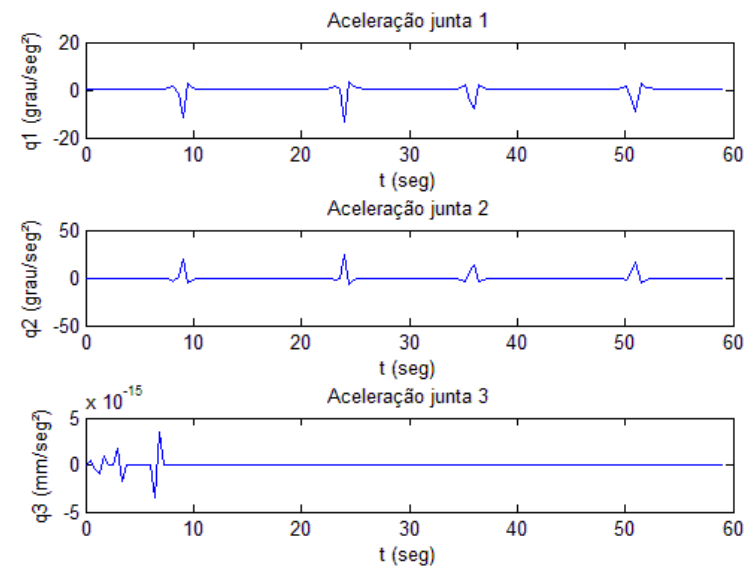

Figura 8: Acelerações de cada junta.
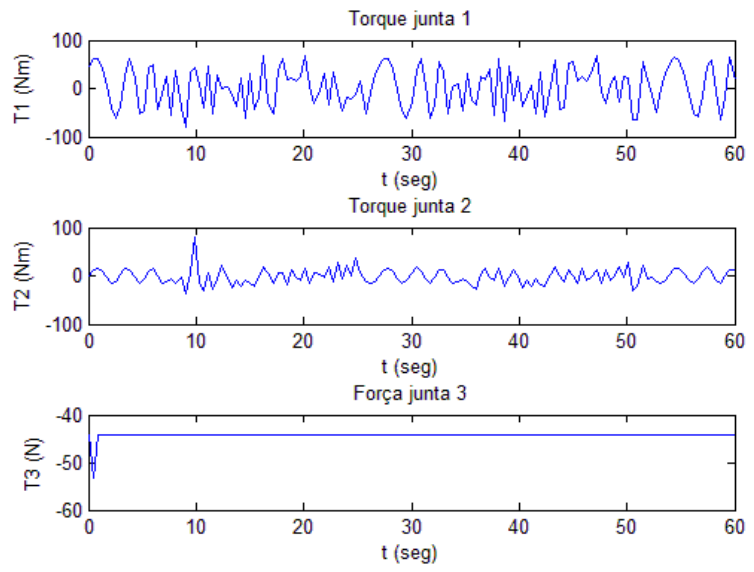

Figura 9: Torques de cada junta. 


\section{Conclusões}

Pode-se extrair, como principal conclusão deste trabalho, que a modelagem dinâmica não é tão simples de ser realizada, pois envolvem várias áreas de conhecimentos, como física, ciências dos materiais, eletrônica, controle, processamento de sinais, programação e manufatura [4].

O proposito desse trabalho foi realizado com êxito, que é a modelagem matemática e a simulação da dinâmica do robô.

A ideia principal para a modelagem da dinâmica é realizar a seleção do controlador e planejador de trajetória que serão abordados em trabalhos futuros.

\section{Agradecimentos}

Os autores agradecem à coordenação do CENTAURO pelo espaço cedido para a montagem do robô.

\section{Referências}

[1] A. Barrientos, Fundamentos de Robótica, Mcgraw-Hill, 1ª ed., Madrid, 1997.

[2] J. J. Craig, Introduction to Robotics, 3rd Edition, Pearson Education, Inc, 2005.

[3] J. J. Craig, Robótica. $3^{\text {a }}$ edição. São Paulo: Editora Pearson, 2012.

[4] I.A. Gervini, L. A. P. Silva, V. S. Rosa e S. C. P. Gomes, Um Estudo Experimental Sobre a Dinâmica de Robôs-Manipuladores do Tipo SCARA. CONEM, 2002.

[5] S. B. Niku, Introduction to robots: analysis, control, applications. John Wiley and Sons, 2011.

[6] P. Eduardo, M. Odair, A. C. Valdiero, "Simulação computacional da não linearidade de folga nas juntas de Manipuladores Robóticos", DINCON, 2010.

[7] V. F. Romano, Robótica Industrial: Aplicações na indústria de manufatura e de processos, Editora Edgard Blucher, São Paulo, 2002.

[8] B. Siciliano, L. Sciavicco, L. Villani and G. Oriolo, Robotics: Modelling, Planning and Control, Springer Publishing Company, Inc, 2009.

[9] M. W. Spong, S. Hutchinson and M. Vidyasagar, Robot Modeling and Control, JohnWiley and Sons, 2006.

[10] L. W. Tsai, Robot analysis: the mechanics of serial and parallel manipulators. John Wiley and Sons, 1999. 